\section{Flavin Transformation by Bacteria}

From a lactoflavin solution which had become blue-fluorescent, a bacterial species has been isolated capable of changing the usual green fluorescence of the flavin solution, and of developing a blue fluorescence. When a very small amount of these bacteria, taken from agar, is put into each of two tubes, one containing aqueous flavin solution, the other only water, the following observations can be made :

1. The green fluorescence of flavin often disappears in about an hour, due to reduction, and may be recovered by shaking with air.

2. In any case the intensity of the green fluorescence becomes gradually less. At the same time a blue fluorescence develops in the solution. The final disappearance of flavin takes about 12 hours with fresh bacteria and $0.6 \gamma$ per c.c. of lactoflavin. More than $3 \gamma$ per c.c. of lactoflavin in the solution is toxic, and no change occurs.

3. The tube containing water and bacteria, but no flavin, does not develop a blue fluorescence. No visible growth occurs in either tube.

4. A tube containing the same amount of flavin under sterile conditions continues to fluoresce green indefinitely.

Brewers' yeast and Clostridium acetobutylicum, both of which contain flavin, do not effect a similar change in flavin solutions; nor does Mycoderma cerevisiae. The bacteria concerned, after drying, give an alcoholic extract showing no green (flavin) fluorescence, but only blue.

The blue-fluorescing substance, either extracted from the bacteria or formed in a flavin solution by a small amount of bacteria, is extractable by chloroform. It may be extracted from chloroform by alkaline water. The blue fluorescence has the same intensity from $p \mathrm{H} 12$ to $p \mathrm{H} 5$, but disappears in more acid solutions. It is not affected by hydrosulphite, or by bromine.

The organism is a Gram-negative rod, occurring in pairs (diplo), and apparently non-spore-forming : a possible relationship to Coli bacteria is being investigated.

The nature of the blue-fluorescing substance, and of its apparent production from flavin by this and other organisms, is being studied. The wide distribution of lactoflavin in Nature, and the existence of a related, blue-fluorescing substance (lumichrome), give these observations special significance.

L. Bradley Petr.

(Overseas Scholar (Canada) of

the Royal Commission for the Exhibition of 1851.)

Biochemical Institute,

Stockholm, Sweden.

Nov. 15.

\section{Cosmic Radiation and Stellar Evolution}

IN connexion with the recent hypotheses ${ }^{1}$ that some of the components of the cosmic rays are ions, it may be noted that the emission of high speed ions from stars would reduce their mass by the same amount as if these ions had been annihilated in the manner suggested by Jeans. The emission of a proton from a star represents the same loss of stellar mass as the transformation of a proton and an electron into a quantum of ultra $\gamma$-radiation.

Thus the emission of cosmic radiation in the form of heavy ions from stars may reconcile the theory of stellar evolution suggested by Eddington's mass luminosity law and the Russell diagram (which seems to require stellar lives of the order only possible if we assume an Einstein-de Sitter universe with a time scale of $10^{12}$ years), and the Friedmann-Lemaittre cosmology with an expanding universe, which suggests that the age of the stars is of the order $10^{10}$ years ; for the emission of heavy ions in such intensity as is indicated by the eosmic ray ionisation observed at high altitudes suggests that stellar mass may decrease appreciably during $10^{10}$ years, since in addition to the loss of mass due to the emission of heat and light radiation, there is the decrease due to the actual ejection of stellar ions probably of high mass.

The process of stellar evolution in the downward direction of the Russell diagram would thus suggest (if the short time scale of the expanding universe is adopted) that the cosmic ray ions are mainly emitted from the heavier stars; and by main sequence stars in passing down the sequence. According to this suggestion, the low mass of the white dwarfs (which are usually assumed to represent the final stage of stellar evolution) shows, therefore, that cosmic rays are entirely emitted from the younger stars, and it is probable, therefore, that the white dwarfs emit practically no radiation in the form of cosmic rays.

Department of Physics,

H. J. WALKE.

Washington Singer Laboratories, University College, Exeter.

${ }^{1}$ Blackett, International Conference on Physics. 1934. Compton and Stephenson, Phys. Rev., 45, 441; 1934.

\section{Formulæ and Equations in Nuclear Chemistry}

IN the advance proofs of the International Conference on Physics, held in London and Cambridge in October 1934, and in other recent publications, the Italian authors write the mass-number and atomic number of the element on the right; for example, $\mathrm{He}_{2}{ }^{4}, \mathrm{Cl}_{17}{ }^{35}$; the English authors write them diagonally, for example, ${ }_{2} \mathrm{He}^{4},{ }_{17} \mathrm{Cl}^{35}$, and the French authors on the left, thus, ${ }_{2}^{4} \mathrm{He},{ }_{17}^{35} \mathrm{Cl}$. When dealing with molecules it is essential to leave a space on the right in which to indicate the number of atoms as in the English formulæ, $\mathrm{H}_{2} \mathrm{O}, \mathrm{Cl}_{2}$, or the French formulæ, $\mathrm{H}^{2} \mathrm{O}, \mathrm{Cl}^{2}$, etc. The Italian scheme blocks both positions and cannot be used by chemists; the English scheme cannot be used by French chemists, whereas the French scheme is convenient for all nationalities and might with advantage be adopted internationally. It has the incidental advantage that, when the numbers are printed vertically above one another, and are not staggered as in the Italian scheme, it is particularly easy to see by subtraction the number of neutrons in the nucleus.

T. M. Lowny.

University Chemical Laboratory, Cambridge.

Dec. 12.

\section{A New Magnetic Alloy with very Large Coercitive Force}

WHILE investigating the magnetic properties of metallic neodymium containing about 7 per cent of iron (the sample was kindly lent to us by Prof. Hopkins of Urbana, Ill.) we found that this material is strongly ferromagnetic. Its specific magnetisation 